Epid

\title{
The association between a genetic risk score for allergy and the risk of developing allergies in childhood-Results of the WHISTLER cohort
}

\author{
Ali Arabkhazaeli $^{1}$ | Fariba Ahmadizar ${ }^{1}$ | Maarten Leusink ${ }^{1,2}$ | Hubertus G. M. Arets $^{3}$ | \\ Jan A. M. Raaijmakers ${ }^{1, \dagger}$ | Cuno S. P. M. Uiterwaal ${ }^{2}$ | Cornelis K. van der Ent ${ }^{3}$ | \\ Anke-Hilse Maitland-van der Zee ${ }^{1,4}$ (D) | Susanne J. H. Vijverberg ${ }^{1,4}$ (i)
}

${ }^{1}$ Division of Pharmacoepidemiology \& Clinical Pharmacology, Faculty of Science, Utrecht Institute for Pharmaceutical Sciences, Utrecht, The Netherlands

${ }^{2}$ Julius Center for Health Sciences and Primary Care, University Medical Center Utrecht, Utrecht, The Netherlands

${ }^{3}$ Department of Pediatric

Pulmonology, WKZ, University Medical Centre Utrecht, Utrecht, The Netherlands

${ }^{4}$ Department of Respiratory

Medicine, Academic Medical Center

(AMC), University of Amsterdam (UvA),

Amsterdam, The Netherlands

\section{Correspondence}

Susanne Vijverberg, Department of

Respiratory Disease, Academic Medical Centre (AMC), University of Amsterdam, Amsterdam,

The Netherlands.

Email: s.j.vijverberg@amc.uva.nl

\begin{abstract}
Background: Several genetic variants have been associated with the susceptibility to allergic disease in adults, but it remains unclear whether these genetic variants are also associated with the onset of allergic disease early in life. The aim of this study was to develop a genetic risk score (GRS) for allergy based on findings in adults and study its predictive capacity for allergy in children.

Methods: A GRS was constructed based on 10 SNPs previously associated with allergies in adults. The GRS was tested in children who participated in a population-based newborn cohort (WHISTLER) and were followed from birth to school age. Logistic regression analysis was used to study the association between the GRS and the parental-reported allergies at age 5 (based on a reported allergy to $\geq 1$ of the following allergens: pollen, house dust mites, or pets). A Cox regression model was used to study the association between GRS and a physician-diagnosed allergy during follow-up (allergic conjunctivitis, allergic rhinitis, and eczema/dermatitis). Cohen's kappa coefficient was calculated to study the agreement between physician-diagnosed allergy and parental-reported allergy at age 5 .

Results: The GRS was significantly associated with parental-reported allergy (odds ratio: 15.9, 95\% confidence interval $(\mathrm{Cl}): 1.07-233.73)$ at age 5, as well as with a physician-diagnosed allergy during follow-up (hazard ratio: $1.89,95 \% \mathrm{Cl}: 1.05-3.41$ ). The overall agreement between physician-diagnosed and parental-reported allergies was $70.5 \%$ (kappa: 0.10, 95\% Cl: 0.03-0.18).

Conclusions: An adult-derived GRS for allergy predicts the risk of developing allergies in childhood.
\end{abstract}

\section{KEYWORDS}

allergy, birth cohort, genetics, risk score

\section{$1 \mid$ INTRODUCTION}

Allergic diseases are characterized by hypersensitivity to normally innocuous antigens. The prevalence of allergic disease has increased

${ }^{\dagger}$ Deceased. globally in the last few decades. More than a third of the world population is affected by allergies; this causes a significant economic burden. ${ }^{1}$ The development of allergic diseases is complex and not fully understood; however, it has been associated with both environmental and genetic components. ${ }^{2}$ Several linkage analyses and candidate gene association studies have previously identified genetic 
variants associated with allergic diseases, such as atopic dermatitis (eg, COL6A5, ${ }^{3}$ FLG, ${ }^{4}$ TLR9, ${ }^{5}$ IL $13,{ }^{6}$ SPINK5, ${ }^{7}$ CMA $1,{ }^{8}$ IL4RA, ${ }^{9}$ and RANTES ${ }^{10}$ ) and allergic rhinitis (eg, FLG, ${ }^{11}$ S100A7, ${ }^{12}$ HDC, ${ }^{13}$ IL13, ${ }^{14}$ $I L 6^{15}$ and $\left.T L R 7^{16}\right) .{ }^{1} \mathrm{~A}$ meta-analysis of genomewide association studies (GWAS) in 53862 individuals has identified 16 shared genomewide loci significantly associated with self-reported pollen allergy, dust mite allergy, and cat allergy. ${ }^{17}$ Of these loci, 8 had previously been associated with asthma. Genetic variants that were found to be associated with self-reported allergy included loci encoded in the 4p14 region near TLR1 (Toll-like receptor 1), TLR6 (Toll-like receptor 6), and TLR10 (Toll-like receptor 10). Those genes encode patternrecognition receptors that play a role in recognizing external molecules and are involved in innate immunity and immune responses. Genetic variation in TLR6 has also been shown to increase the risk of high total IgE in children having two or more older siblings. ${ }^{18}$ Other associations in the GWAS meta-analysis included loci in genes involving in the presentation of intracellular peptides to $\mathrm{T}$ cells, genes involved in the initiation of skin immune responses, $\mathrm{TH} 2$ differentiation (BCL6, IL2/ADAD1), cell-cell adhesion (LPP), and genes involved in the regulation of $\mathrm{TH} 2$-mediated inflammation and mucus production in allergic airway disease. ${ }^{17}$

To summarize the combined effect of genetic variants on an outcome, such as the susceptibility of allergies, a genetic risk score (GRS) can be constructed. A GRS-sometimes called allele scores, gene scores, or genotype scores-comprises of a quantitative score of previously associated SNPs. ${ }^{19}$

Although most of the genetics studies for allergies were performed in adults, the collective dataset suggests an important genetic basis for allergic diseases. This raises the question whether genetic risk factors associated with allergic diseases in adults are predictive for the development of allergic diseases in children as well. In this current study, we aimed to develop a GRS for the onset of allergy in children based on findings in adults, to study whether genetic risk factors for allergy later in life are also predictive for the risk of developing allergies early in life.

\section{2 | METHODS}

\section{1 | Study population}

We analyzed data collected in the Wheezing Illnesses Study Leidsche Rijn (WHISTLER) cohort, a prospective population-based birth cohort study in the Netherlands, which studies determinants and prediction of wheezing illnesses. Study design and rationale of WHISTLER have been described in detail elsewhere. ${ }^{20}$ Briefly, healthy neonates and infants born in a newly developed residential area near the city of Utrecht in the Netherlands (ie, Leidsche Rijn) were invited by telephone to participate in this study before the age of 2 months. Exclusion criteria were gestational age $<36$ weeks, major congenital abnormalities, and neonatal respiratory disease. During an extended first consultation, an "Individual Health Profile" (IHP) was compiled and buccal swabs were obtained for DNA extraction. Children were followed throughout childhood. At the age of 5 years, children were invited for a second study visit to the local outpatient clinic, in which information about general health, allergic symptoms, asthma and respiratory symptoms, medication use, preand postnatal risk factors was obtained by questionnaires. Children were invited for a third study visit to the local outpatient clinic at the age of 8 years. During the total follow-up, information on physician diagnoses, primary care consultations, and medication prescriptions for respiratory symptoms was collected. The medical ethics committee of the University Medical Center Utrecht, Utrecht, the Netherlands, approved the WHISTLER cohort study. Written informed consent was obtained from the parents and included permission to use relevant child health information obtained from general practitioners.

\section{2 | Study design}

In this population-based birth cohort, we assessed the association between a genetic risk score for allergy susceptibility based on genetic variants associated with allergy in adults and allergies (parental-reported and doctor-diagnosed) in children.

\section{3 | Genotyping and quality control}

Genomic DNA was extracted from buccal cells of infants using the QIAamp DNA blood mini kit (Qiagen, GmbH, Hilden, Germany), and concentration was determined using PicoGreen (Molecular Probes, Thermofisher scientific, Waltham, MA, USA). ${ }^{20}$ Genotyping was performed using the Infinium HumanExome chip (Illumina, San Diego, CA), version 1.1, which contains 242902 variants. ${ }^{21}$ Genotypes obtained from GenomeStudio were used for quality control (QC), and PLINK v1.07 was used for the downstream process. Sample QC was performed on common SNPs (MAF $\geq 5 \%$ ) of high quality (missingness $<1 \%$, Hardy-Weinberg equilibrium $P>1 \mathrm{e}-4$, and LD-pruned to leave no pairs with $r^{2}>.2$ ). We removed samples based on heterozygosity, keeping samples within 4 standard deviations. European ancestry was verified using EIGENSTRAT, ${ }^{22}$ and non-European samples were excluded for further analysis. Identity-by-descent estimates from PLINK were used to identify siblings or otherwise related children (pi-hat $>0.2$ ), one of which was randomly excluded. As the calling algorithms in GenomeStudio are not designed for rare SNPs, genotypes from $z \mathrm{Call}^{23}$ were used in subsequent analyses, where we excluded SNPs with a call rate less than $95 \%$ and a Hardy-Weinberg equilibrium $P$-value $<1 \mathrm{e}-6$.

\subsection{Genetic risk score}

A GRS was constructed based on SNPs previously associated with allergies in adults in a GWAS meta-analysis by Hinds et $\mathrm{al}^{17}$ as there were no GWAS available for children. In this meta-analysis, 16 SNPs were found to be associated with at least one of three common self-reported allergy phenotypes (pollen allergy, dust mite allergy, and cat allergy) in two cohorts with a total of 53862 individuals. 
Of the 16 identified shared susceptibility loci with association $P<5 e-8$, reported by Hinds et al, three were available on the exome chip. Moreover, we found a suitable alternative on the chip for seven more SNPs, for which our criterion was that they should have an $r^{2}>.8$. The $r^{2}$ could be regarded as the correlation between the original and the alternative SNP, as a rough measure, with $r^{2}=.8$; in $80 \%$ of the cases, if you have genotyped the original SNP, you will also have information on the correlated SNP. Therefore, we could include 10 SNPs in our GRS. We weighted the SNPs in proportion to their effect size, as these varied considerably between the SNPs. The weighting was based on the natural logarithm of the reported odds ratios (ORs) for the allergy SNPs in the original GWAS. ${ }^{17}$ The GRS was weighted in such a way that an increase in the GRS would be associated with an increase in allergies in the original GWAS. SNPs included in the risk score, as well as effect sizes, effect alleles, references to studies identifying them, and detail on generating GRS can be found in the supplementary materials (Table S1).

\subsection{Outcomes}

1. Parental-reported allergy to pollens, dust mites, and/or pets at age 5. This was based on $\geq 1$ positive answer to one of the following questions in the questionnaire during the study visit at age 5 and age 8: "Is your child allergic to pollen?", "Is your child allergic to house dust mite?", "Is your child allergic to certain pets?"

2. Physician diagnosis of allergic symptoms, using the International Classification of Primary Care (ICPC), ${ }^{24}$ allergic conjunctivitis (ICPC: F71), allergic rhinitis (ICPC: R97), or eczema/dermatitis (ICPC: S87, S88). These data were collected from the primary healthcare records.

\section{6 | Statistical analysis}

Logistic regression analysis was applied to study the association between the GRS and the parental-reported allergies at age 5 and age 8. Odds ratios (ORs), 95\% confidence intervals $(\mathrm{Cl})$, and $p$-values were calculated, and the model was adjusted for age, gender, breastfeeding, and a family history of asthma or allergy. To study the association between the GRS and physician diagnosis of allergic symptoms, a Cox regression analysis was used, as data were available regarding the timing of physician diagnosis of allergic symptoms. Hazard ratios, $95 \% \mathrm{Cl}$, and $P$-values were calculated, and the model was adjusted for age, gender, breastfeeding, and a family history of asthma or allergy. The agreement between parentalreported allergy (to specific allergens) and physician-diagnosed allergy (based on allergic symptoms) was assessed with Cohen's kappa statistics and interpreted using the classification system developed by Landis and $\mathrm{Koch}^{25}: \kappa \leq 0$ indicates poor agreement, $\kappa$ between 0 and 0.20 indicates slight agreement, 0.21-0.40 indicates fair agreement, 0.41-0.60 moderate agreement, $0.61-0.80$ substantial agreement, and $\kappa$ values of 0.81 to 1.00 indicates almost perfect to perfect agreement.

For the analyses, we used several statistical packages: R (version 3.0.2, http://www.R-project.org ), SPSS (version 23.0, IBM SPSS Statistics for Windows, Armonk, NY: IBM Corp), and PLINK (version 1.07).

\section{3 | RESULTS}

\section{1 | Study population}

In total, 2112 children who participated in the WHISTLER study were genotyped, of which 692 were excluded in the genotyping QC process, leaving 1420 children for our analyses. The high exclusion rate of the genetic samples was mainly due to large amount of related individuals in the WHISTLER cohort. Physician diagnosis data during follow-up were available for 1330 children, and parental-reported data at ages 5 and 8 were available for 465 and 163 children, respectively (Figure 1 and Table S2). Characteristics of the study population are shown in Table 1. The median age of our study population was 7.0 years (interquartile range (IQR): 4.0-9.0), and half of the children were girls (50.8\%). The mean genetic risk score of the children in the study was $0.7 \pm 0.28$.

\section{2 | GRS and parental-reported allergies}

At age 5, parents of 28 of the 456 children (6.0\%) reported that their child suffered from an allergy. The GRS was statistically significantly associated with parental-reported allergy in this population (OR: 15.9, 95\% Cl: 1.07-233.73) (Table 2). At age 8, 16 of 163 children (11.2\%) suffered from allergies based on parental-reported questionnaire. The GRS was not significantly associated with parental-reported allergies in this population.

\section{3 | GRS and physician-diagnosed allergy}

We retrospectively assessed the time to a physician-diagnosed allergy in the WHISTLER population. The median follow-up time was

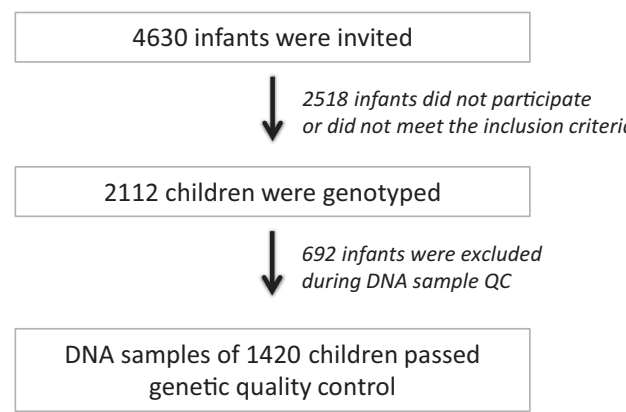

90 children did not have physician data available

Count of parental-reported data available at age 5
1330 children had data on physician diagnosis available
465 children had parental-reported data available at age 5

Count of parental-reported data available at age 8

163 children had parental-reported data available at age 8

FIGURE 1 Overview of the recruitment and inclusion of children from the Wheezing Illnesses Study Leidsche Rijn (WHISTLER) project 
TABLE 1 Characteristics of the study population

\begin{tabular}{|c|c|}
\hline Subjects, $n$ & 1330 \\
\hline Age, median (IQR) & $7.0(4.0-9.0)$ \\
\hline Dutch ethnicity, ${ }^{a} \mathrm{n}(\%)$ & $471 / 569(82.8)$ \\
\hline Gender (female), n (\%) & $675 / 1330(50.8)$ \\
\hline Follow-up duration in $y r,{ }^{b}$ median (IQR) & $3.8(5.1)$ \\
\hline Physician-diagnosed asthma, n (\%) & $110 / 1330(7.1)$ \\
\hline $\begin{array}{l}\text { Family history of allergies (father or mother), } \\
\mathrm{n}(\%)\end{array}$ & $842 / 1150(73.2)$ \\
\hline $\begin{array}{l}\text { Family history of asthma (father or mother), } \\
\mathrm{n}(\%)\end{array}$ & $168 / 1098(15.3)$ \\
\hline Genetic allergies risk score, mean (SD) & $0.7(0.2)$ \\
\hline
\end{tabular}

$\mathrm{IQR}$, interquartile range; SD, standard deviation.

a Both parents Dutch.

${ }^{b}$ From birth to data extraction dates.

TAB LE 2 Association between genetic risk score for allergy and risk of parental-reported allergy at age 5

\begin{tabular}{|lll} 
& $\begin{array}{l}\text { Crude OR (95\% Cl), } \\
\text { P-value }\end{array}$ & $\begin{array}{l}\text { Adjusted OR (95\% } \\
\text { Cl), }{ }^{\text {a }} \text { P-value }\end{array}$ \\
\hline $\begin{array}{l}\text { Parental-reported } \\
\text { allergy }\end{array}$ & $\begin{array}{l}9.47(1.04-86.45), \\
15.88(1.07-233.73),\end{array}$ & .044 \\
\hline
\end{tabular}

GRS, genetic risk score; OR, odds ratio; $\mathrm{Cl}$, confidence interval; $\mathrm{RA}$, parental-reported allergy to pollen, house mites or pets.

${ }^{a}$ Adjusted for age, gender, breastfeeding, family history of asthma, and family history of allergies.

TAB LE 3 Association between genetic risk score for allergy and risk of physician diagnosis of allergies during childhood

\begin{tabular}{lll} 
& $\begin{array}{l}\text { Crude HR (95\% CI), } \\
\text { P-value }\end{array}$ & $\begin{array}{l}\text { Adjusted HR (95\% } \\
\text { CI), }{ }^{\text {a }} \text { P-value }\end{array}$ \\
\hline $\begin{array}{c}\text { Physician- } \\
\text { diagnosed allergy }\end{array}$ & 1.79 (1.06-3.02), .030 & 1.89 (1.05-3.41), .035 \\
\hline
\end{tabular}

GRS, genetic risk score; $\mathrm{HR}$, hazard ratio; $\mathrm{Cl}$, confidence interval.

${ }^{a}$ Adjusted for age, gender, breastfeeding, family history of asthma, and family history of allergies.

3.6 years (IQR: 1.5-6.7 years) starting from birth to the occurrence of any allergies or extraction date. The allergy GRS was statistically significantly associated with the risk of a physician diagnosis of allergies (hazard ratio (HR):1.89, 95\% Cl: 1.05-3.41) (Table 3). Children with higher allergy GRS had a higher chance to be diagnosed with allergic conjunctivitis, rhinitis, or dermatitis during the follow-up. The effect of GRS on diagnosed allergic conjunctivitis or rhinitis separately was not statistically significant $(\mathrm{HR}=1.90,95 \% \mathrm{Cl}: 0.6-5.9)$.

\section{4 | The agreement between parental-reported and physician-diagnosed allergies}

For 455 children, data were available to assess the agreement between diagnosed allergies (allergic conjunctivitis, allergic rhinitis, or dermatitis at age 5 years) and parental-reported allergy to pollen, house mites, or pets at age 5. Overall agreement was 70.5\% (Table 4); kappa was 0.10 (95\% Cl: 0.03-0.18), which indicated "slight agreement." There was also a slight agreement (62.9\%) at age 8 (Table 5) with a kappa of $0.14(95 \%$ $\mathrm{Cl}$ : 0.02-0.26). When dermatitis would be left out of the physiciandiagnosed allergy outcome, the agreement was $94.9 \%$ (Table S3) with kappa statistics of 0.49 at 5 years old and $89.1 \%$ (Table S4) with kappa of 0.47 at 8 years old, which indicate "moderate agreement."

\section{DISCUSSION}

This study showed that genetic variants previously associated with allergies in adults are also predictive of the onset of allergies in children. Development of allergic diseases is complex. Both environmental factors such as diet, family size, education level in the household, specific infections, exposures to non-pathogenic microorganisms, living in urban settings and regions with low exposure to ultraviolet radiation and low humidity, ${ }^{26}$ and genetic factors have been shown to be involved. ${ }^{2}$ A century ago, it was already shown that there is a heritable component in the risk of developing allergies. ${ }^{27}$ More recently, large GWAS have identified genetic variants associated with allergic sensitization and allergic symptoms. ${ }^{17,28}$ Here, we showed that combining established genetic risk factors in a predictive risk score can be used to predict the onset of allergy in children.

That a GRS might be a useful tool to predict allergies has also been shown by Bønnelykke et al. ${ }^{28}$ They constructed a GRS based on 10 genomewide significant loci based on a different, somewhat smaller, reference GWAS ${ }^{29}$ including 10 studies (birth cohorts and populationbased cohorts). They tested the predictive capacity of the GRS for allergic sensitization and allergic rhinitis in a population-based study ( $n=9258$, mostly adults) and showed that a higher GRS score indicated a higher prevalence of sensitization. In our study, we applied a similar approach but restricted the GRS to genetic variants associated with allergy in adults and tested the GRS in an independent birth cohort to assess the predictive value for allergies early in life. Nevertheless, there is a large overlap in the loci studied; of the ten loci Bønnelykke et al studied, 5 loci were also included in our study. A complete report of similarity and differences of the two mentioned reference GWAS was published by Bønnelykke et al. ${ }^{29}$

The comparability of different genetic allergy studies is limited due to the application of different definitions of allergy ${ }^{1}$; some studies focus on allergic symptoms and use self-reported allergy, and others focus on physician diagnosis of allergy or measures of allergic sensitization such as elevated levels of allergen-specific lgE and/or a positive skin prick test. Although allergic sensitization can be measured more objectively, it does not provide proof that the sensitization will also cause allergic symptoms. In this study, we assessed allergic symptoms, focusing on self/parental-reported, as well as physician-diagnosed allergies. As both outcomes might measure different aspects of allergic diseases, we performed agreement analyses to see whether these outcomes identified different individuals. We found a slight statistically significant agreement between reported allergy to pollen, house mites, or pets and diagnosed allergies. We assume that one of the reasons 
TABLE 4 The agreement between parental-reported and physician-diagnosed allergies at age $5 \mathrm{y}$

\begin{tabular}{|c|c|c|c|c|}
\hline & & & \multicolumn{2}{|c|}{$\begin{array}{l}\text { Physician-diagnosed } \\
\text { allergic symptoms at } \\
\text { age } 5 \text { y }\end{array}$} \\
\hline & & & No & Yes \\
\hline \multirow{4}{*}{$\begin{array}{l}\text { Parental-reported } \\
\text { allergy to pollen, } \\
\text { house dust mite } \\
\text { or pets at age } 5 \mathrm{y}\end{array}$} & \multirow[t]{2}{*}{ No } & Count & 305 & 122 \\
\hline & & $\%$ of Total & $67.0 \%$ & $26.8 \%$ \\
\hline & \multirow[t]{2}{*}{ Yes } & Count & 12 & 16 \\
\hline & & $\%$ of Total & $2.6 \%$ & $3.5 \%$ \\
\hline
\end{tabular}

Kappa $(95 \% \mathrm{Cl})=0.10(0.03-0.18)$.

TABLE 5 The agreement between parental-reported and physician-diagnosed allergies at age $8 \mathrm{y}$

\begin{tabular}{|c|c|c|c|c|}
\hline & & & \multicolumn{2}{|c|}{$\begin{array}{l}\text { Physician-diagnosed } \\
\text { allergic symptoms at } \\
\text { age } 8 \text { y }\end{array}$} \\
\hline & & & No & Yes \\
\hline \multirow{4}{*}{$\begin{array}{l}\text { Parental-reported } \\
\text { allergy to pollen, } \\
\text { house dust mite } \\
\text { or pets at age } 8 \mathrm{y}\end{array}$} & \multirow[t]{2}{*}{ No } & Count & 79 & 48 \\
\hline & & $\%$ of Total & $55.2 \%$ & $33.6 \%$ \\
\hline & \multirow[t]{2}{*}{ Yes } & Count & 5 & 11 \\
\hline & & $\%$ of Total & $3.5 \%$ & $7.7 \%$ \\
\hline
\end{tabular}

$\operatorname{Kappa}(95 \% \mathrm{Cl})=0.14(0.02-0.26)$.

for the lack of agreement is that not all children visit their physician with allergic complaints. Nevertheless, the GRS was associated with parental-reported allergy (at age 5), as well as physician-diagnosed allergy (during follow-up). In summary, an allergy GRS based on SNPs associated with allergies in adults can predict the risk on developing allergies in childhood. As allergy has multiple risk factors including genetic components, we believe that this allergy GRS can be used to develop a prediction model for allergic symptoms.

\section{ORCID}

Anke-Hilse Maitland-van der Zee

http://orcid.org/0000-0002-0414-3442

Susanne J. H. Vijverberg (iD http://orcid.org/0000-0002-4579-4081

\section{REFERENCES}

1. Portelli MA, Hodge E, Sayers I. Genetic risk factors for the development of allergic disease identified by genome-wide association. Clin Exp Allergy. 2015;45:21-31.

2. Thomsen SF, Ulrik CS, Kyvik KO, et al. Genetic and environmental contributions to hay fever among young adult twins. Respir Med. 2006;100:2177-2182.

3. Soderhall C, Marenholz I, Kerscher T, et al. Variants in a novel epidermal collagen gene (COL29A1) are associated with atopic dermatitis. PLoS Biol. 2007;5:e242.

4. Morar N, Cookson WO, Harper JI, Moffatt MF. Filaggrin mutations in children with severe atopic dermatitis. J Invest Dermatol. 2007;127:1667-1672.
5. Novak N, Yu CF, Bussmann C, et al. Putative association of a TLR9 promoter polymorphism with atopic eczema. Allergy. 2007;62: 766-772.

6. Nickel R, Beyer K, Huang SK, Barnes KC, Wahn U. Genetic markers of atopy in infancy: results from the German Multicenter Allergy Study. Clin Exp Allergy. 1999;29(Suppl 4):23-25.

7. Chavanas S, Bodemer C, Rochat A, et al. Mutations in SPINK5, encoding a serine protease inhibitor, cause Netherton syndrome. Nat Genet. 2000;25:141-142.

8. Iwanaga T, McEuen A, Walls AF, et al. Polymorphism of the mast cell chymase gene (CMA1) promoter region: lack of association with asthma but association with serum total immunoglobulin E levels in adult atopic dermatitis. Clin Exp Allergy. 2004;34:1037-1042.

9. Novak N, Kruse S, Kraft S, et al. Dichotomic nature of atopic dermatitis reflected by combined analysis of monocyte immunophenotyping and single nucleotide polymorphisms of the interleukin-4/interleukin-13 receptor gene: the dichotomy of extrinsic and intrinsic atopic dermatitis. J Invest Dermatol. 2002;119:870-875.

10. Tanaka K, Roberts MH, Yamamoto N, Sugiura H, Uehara M, Hopkin JM. Upregulating promoter polymorphisms of RANTES relate to atopic dermatitis. Int J Immunogenet. 2006;33:423-428.

11. Tang XF, Tang HY, Sun LD, et al. Genetic variant rs4982958 at $14 q 11.2$ is associated with allergic rhinitis in a Chinese Han population running title: $14 q 11.2$ is a susceptibility locus for allergic rhinitis. J Investig Allergol Clin Immunol. 2012;22:55-62.

12. Bryborn M, Hallden C, Sall T, Adner M, Cardell LO. Comprehensive evaluation of genetic variation in S100A7 suggests an association with the occurrence of allergic rhinitis. Respir Res. 2008;9:29.

13. Gervasini G,AgundezJA, Garcia-Menaya J, etal.Variability of the L-Histidine decarboxylase gene in allergic rhinitis. Allergy. 2010;65:1576-1584.

14. Li JY, Zhang Y, Lin XP, et al. Association between DNA hypomethylation at IL13 gene and allergic rhinitis in house dust mite-sensitized subjects. Clin Exp Allergy. 2016;46:298-307.

15. Nasiri R, Movahedi M, Amirzargar AA, et al. Association of interleukin 6 single nucleotide polymorphisms with allergic rhinitis. Int J Pediatr Otorhinolaryngol. 2014;78:1426-1429.

16. Nilsson D, Andiappan AK, Hallden C, et al. Toll-like receptor gene polymorphisms are associated with allergic rhinitis: a case control study. BMC Med Genet. 2012;13:66.

17. Hinds DA, McMahon G, Kiefer AK, et al. A genome-wide association meta-analysis of self-reported allergy identifies shared and allergyspecific susceptibility loci. Nat Genet. 2013;45:907-911.

18. Reijmerink NE, Kerkhof M, Bottema RW, et al. Toll-like receptors and microbial exposure: gene-gene and gene-environment interaction in the development of atopy. Eur Respir J. 2011;38:833-840.

19. Burgess S, Thompson SG. Use of allele scores as instrumental variables for Mendelian randomization. Int J Epidemiol. 2013;42:1134-1144.

20. Katier N, Uiterwaal CS, de Jong BM, et al. The Wheezing Illnesses Study Leidsche Rijn (WHISTLER): rationale and design. Eur J Epidemiol. 2004;19:895-903.

21. Anonymous Exome Chip Design - Genome Analysis Wiki; 2014.

22. Price AL, Patterson NJ, Plenge RM, Weinblatt ME, Shadick NA, Reich D. Principal components analysis corrects for stratification in genome-wide association studies. Nat Genet. 2006;38:904-909.

23. Goldstein JI, Crenshaw A, Carey J, et al. zCall: a rare variant caller for array-based genotyping: genetics and population analysis. Bioinformatics. 2012;28:2543-2545.

24. Verbeke M, Schrans D, Deroose S, De Maeseneer J. The International Classification of Primary Care (ICPC-2): an essential tool in the EPR of the GP. Stud Health Technol Inform. 2006;124:809-814.

25. Landis JR, Koch GG. The measurement of observer agreement for categorical data. Biometrics. 1977;33:159-174.

26. WeidingerS, NovakN. Atopic dermatitis. Lancet. 2016;387:1109-1122.

27. Cooke RA, Vander Veer Jr A. Human sensitization. J Immunol. 1916:1:201-305 
28. Bonnelykke K, Matheson MC, Pers TH, et al. Meta-analysis of genome-wide association studies identifies ten loci influencing allergic sensitization. Nat Genet. 2013;45:902-906.

29. Bonnelykke K, Sparks R, Waage J, Milner JD. Genetics of allergy and allergic sensitization: common variants, rare mutations. Curr Opin Immunol. 2015;36:115-126.

\section{SUPPORTING INFORMATION}

Additional Supporting Information may be found online in the supporting information tab for this article.
How to cite this article: Arabkhazaeli A, Ahmadizar F, Leusink $M$, et al. The association between a genetic risk score for allergy and the risk of developing allergies in childhoodResults of the WHISTLER cohort. Pediatr Allergy Immunol. 2018;29:72-77. https://doi.org/10.1111/pai.12824 